\title{
Garantia da qualidade aplicada à produção de radiofármacos
}

\author{
Elaine Bortoleti de Araújo*, Tatiana Lavinas, Maria Tereza Colturato, Jair Mengatti
}

Centro de Radiofarmácia, Instituto de Pesquisas Energéticas e Nucleares, IPEN-CNEN

*Correspondência:

E. B. Araújo

Centro de Radiofarmácia

Instituto de Pesquisas Energéticas e

Nucleares- IPEN-CNEN

Av. Professor Lineu Prestes, 2242

Cidade Universitária

05508-000 - São Paulo -SP, Brasil

E-mail: ebaraujo@ipen.br
Radiofármacos são produzidos e distribuidos no Brasil há mais de 40 anos pelos Institutos da Comissão Nacional de Energia Nuclear (CNEN), particularmente o Instituto de Pesquisas Energéticas e Nucleares (IPEN), para uso em procedimentos diagnósticos e terapêticos em Medicina Nuclear. Alguns aspectos da produção, distribuição e utilização dos radiofármacos são bastante particulares, diferenciando-se dos fármacos convencionais, tornando necessário estabelecer regulamentação específica para tais produtos. Neste sentido, existem orientações da Organização Mundial de Saúde (OMS) bem como regulamentações de órgãos sanitários de diversos países que já fazem distinção aos radiofármacos nas legislações específicas.

\section{INTRODUÇÃO}

Radiofármacos podem ser definidos simplesmente como substratos que contêm um átomo radioativo em sua estrutura, podendo ser considerados como vetores que apresentam certa especificidade por algum órgão ou uma função fisiológica ou fisiopatológica. Por sua forma farmacêutica, quantidade e qualidade da radiação emitida, podem ser utilizados com finalidade diagnóstica ou terapêutica, qualquer que seja a via de administração empregada (Sorenson, Phelps, 1987; Shung, Smith, Tsui, 1992; Chandra, 1992; Zolle, 2007).

Conforme descrito pela Organização Mundial de Saúde (OMS), radiofármacos são produtos farmacêuticos que podem ser classificados em quatro categorias (World Health Organization, 2004):

(a) Produtos radioativos prontos para uso;

(b) Geradores de radionuclídeos;

(c) Componentes não radioativos (reagentes liofilizados) para preparação de compostos marcados com elementos radioativos (geralmente o eluato de um gera- dor de radionuclídeo);

(d) Precursores utilizados para marcação de outras substâncias antes da administração (ex. amostras provenientes dos pacientes, como células sanguíneas).

O termo "droga radioativa" conforme definido no CFR 21 do FDA ("Code of Federal Regulation n' 21 Food and Drug Administration") é aplicado a substâncias que exibem desintegração espontânea de um núcleo instável (radioativo) com a emissão de partículas ou fótons e inclui os reagentes não radioativos ou geradores de radionuclídeos destinados à preparação de tais substâncias, mas não inclui drogas que contêm apenas quantidades traços de radionuclídeos de ocorrência natural (CFR 21, Part 315).

A ação da maioria dos radiofármacos é derivada de dois componentes: um componente não radioativo (um carregador ou ligante) e um componente radioativo (ou radionuclídeo). Os ligantes ou carregadores dirigem o radionuclídeo a um órgão específico ou processo, onde o componente radionuclídico pode ser detectado. Os meca- 
nismos que promovem a ligação do radiofármaco ao sítio alvo podem ser diversos, envolvendo desde uma simples perfusão sanguínea do composto pelos órgãos de interesse, até a ligação a receptores celulares específicos ou participação em uma via metabólica ou processo bioquímico. Esta particularidade dos radiofármacos distingue a técnica diagnóstica da Medicina Nuclear de outras técnicas como a Ressonância Magnética ou Tomografia convencional que se limitam, na maioria das vezes, a obter imagens da estrutura anatômica, sem uma correlação funcional (Sorenson, Phelps, 1987; Shung, Smith, Tsui, 1992; Chandra, 1992).

A Medicina Nuclear pode ser definida como a especialidade médica que utiliza as propriedades nucleares de compostos radioativos para realizar avaliações diagnósticas das condições anatômicas ou fisiológicas, tratamentos terapêuticos e pesquisas médicas. Um aspecto único da Medicina Nuclear é a sensibilidade elevada para detectar alterações na função ou morfologia de um determinado órgão, fazendo uso dos radiofármacos (Sorenson, Phelps, 1987; Shung, Smith, Tsui, 1992; Chandra, 1992; Zolle, 2007).

Os ligantes empregados na elaboração de radiofármacos incluem compostos orgânicos, inorgânicos e biomoléculas (peptídeos, anticorpos monoclonais e seus fragmentos e oligonucleotídeos). Desta forma os radiofármacos são classificados pelo FDA como droga ou produto biológico (CFR 21, Part 315).

Os radiofármacos podem ser administrados oralmente ou por inalação, mas a grande maioria é administrada por meio de injeção intravenosa.

A grande aplicação dos radiofármacos está em Medicina Nuclear diagnóstica, representando cerca de 95\% dos procedimentos em Medicina Nuclear. Nos últimos anos, entretanto, tem crescido consideravelmente a aplicação dos radiofármacos em procedimentos terapêuticos, envolvendo desde a simples administração de solução de iodeto de sódio (iodo-131) para terapia de câncer de tireóide e hipertireoidismo, até o uso de peptídeos e anticorpos monoclonais específicos, como o anticorpo anti-CD-20 marcado com elementos radioativos emissores beta (itrio90, lutécio-177 e iodo-131), empregado na terapia de linfoma do tipo não-Hodgkin, numa modalidade terapêutica denominada Radioimunoterapia (Araújo et al., 2004; Araújo et al., 2005; Akanji et al., 2005; Nagamati et al., 2005; Caldeira Filho et al., 2005; Caldeira Filho et al., 2006).

Nos radiofármacos para terapia o radionuclídeo é geralmente um elemento emissor de partículas (freqüentemente do tipo beta menos ou négatron), com energia para promover a destruição de células e alcance relativamente curto, evitando a irradiação de tecidos sadios situados ao redor do tecido alvo. Os radiofármacos para terapia são utilizados para promover uma alteração em uma estrutura alvo ou um processo de doença, apresentando aplicação mais freqüente na terapia do câncer (destruição do tecido tumoral) e também em radiosinovectomia (tratamento de artrite reumatóide a partir da aplicação de radiofármacos na cavidade sinovial) (Couto et al., 2006A; Couto et al., 2006B).

Os radiofármacos para diagnóstico, por sua vez, são utilizados para adquirir imagens ou identificar uma estrutura interna ou um processo de doença. O componente de imagem geralmente é um elemento radioativo de meia vida curta que emite fótons a partir do decaimento radioativo com suficiente energia para penetrar a massa tecidual do paciente. Os fótons emitidos são detectados por aparatos especiais que geram imagens (câmaras gama ou tomógrafos para emissão de fótons - SPECT ("Single Photon Emission Computer Tomography") ou para emissão de pósitrons - PET ("Positron Emission Tomography") (Sorenson, Phelps, 1987; Shung, Smith, Tsui, 1992; Chandra, 1992).

O Instituto de Pesquisas Energéticas e Nucleares (IPEN), por meio do seu antigo Departamento de Processamento de Material Radioativo (TP), atual Centro de Radiofarmácia (CR), foi a Instituição pioneira na produção de radioisótopos e radiofármacos no Brasil.

O IPEN é uma autarquia estadual, vinculada à Secretaria de Ciência, Tecnologia e Desenvolvimento Econômico do Governo do Estado de São Paulo, gerido técnica e administrativamente pela Comissão Nacional de Energia Nuclear (CNEN) do Ministério da Ciência e Tecnologia. O IPEN está instalado no campus da Cidade Universitária em São Paulo e é ligado à Universidade de São Paulo por seu programa de pós-graduação.

A produção experimental de radiofármacos, iniciada no IPEN em 1959 com o iodo-131, usado para diagnóstico e terapia de doenças da tireóide, foi fundamental para a viabilização e consolidação da Medicina Nuclear no país.

No início da década de 80 o IPEN estabeleceu um programa de desenvolvimento e iniciou os trabalhos experimentais com molibdênio-99 importado do Canadá para produção de geradores de molibdênio-tecnécio $\left({ }^{99} \mathrm{Mo}-{ }^{99 \mathrm{~m}} \mathrm{Tc}\right)$. Em 1981, iniciou o atendimento a hospitais e clínicas do país com geradores preparados com tecnologia nacional, atendendo uma demanda inicial de 10 geradores por semana. Esta demanda tem crescido anualmente e atingiu em 2006 a marca de 280 geradores distribuídos por semana.

O tecnécio-99m é um dos principais elementos radioativos empregados na elaboração de radiofármacos, sendo utilizado na maior parte dos procedimentos diagnósticos de Medicina Nuclear. Paralelamente ao desenvolvimento do gerador de ${ }^{99} \mathrm{Mo}-{ }^{99 m} \mathrm{Tc}$, inúmeros reagentes liofilizados para marcação com tecnécio-99m foram desenvolvidos e disponibilizados para a classe médica nuclear. Cada 
reagente liofilizado, reagindo com o tecnécio-99m oriundo do gerador de radionuclídeo, origina um radiofármaco com propriedades específicas para o diagnóstico de determinados órgãos e/ou patologias (Araújo et al., 1990; Araújo et al., 1995; Carvalho et al., 1996; Abreu et al., 2006).

Outros radiofármacos foram desenvolvidos, produzidos e distribuídos pelo IPEN, como o cloreto de ${ }^{201} \mathrm{Tl}$, para estudo da viabilidade cardíaca, o citrato de gálio-67 para diagnóstico de tumores específicos e pesquisa de focos de inflamação e infecção, a Metaiodobenzilguanidina marcada com iodo-131 e iodo-123, utilizada, respectivamente, na terapia e diagnóstico de feocromocitomas e neuroblastomas, além do ${ }^{153} \mathrm{Sm}$, que na forma de EDTMP${ }^{153} \mathrm{Sm}$, age como paliativo das dores provenientes de metástases ósseas de câncer de próstata e mama, entre outros (Braga et al., 1993A; Braga et al., 1993B).

Em 1999 foi iniciada a produção e distribuição de Fluordesoxiglicose- ${ }^{18} \mathrm{~F}\left(\mathrm{FDG}-{ }^{18} \mathrm{~F}\right)$ para estudo da viabilidade miocárdica e localização de tumores, com grande aceitação pela classe médica nuclear. $\mathrm{O}^{18} \mathrm{~F}$ foi o primeiro radioisótopo produzido no País, para uso na técnica de PET que permite maior sensibilidade nas imagens obtidas e diagnósticos mais precisos.

A partir de 1995, o IPEN iniciou um programa de nacionalização, com objetivo de passar a produzir parte dos radioisótopos então importados. Esse programa se consolidou com o aumento da potência do Reator IEA-R1, possibilitando a produção local de iodo-131 e samário-153 e com a instalação do ciclotron de $30 \mathrm{MeV}$, responsável pela produção nacional de tálio-201, gálio-67, iodo-123 e flúor18. Em 2006 o CR produziu $100 \%$ da necessidade anual do mercado nacional de gálio-67 e de tálio-201 e cerca de $70 \%$ de iodo-131.

O programa de nacionalização, associado ao desenvolvimento constante de novos produtos, tem propiciado ao IPEN produzir e distribuir para todo o território nacional, diversos produtos radioativos para diagnóstico e tratamentos, entre eles, radioisótopos primários, moléculas marcadas e reagentes liofilizados para pronta marcação com ${ }^{99 m}$ Tc (Araújo et al., 1990; Araújo et al., 1995; Carvalho et al., 1996; Santos et al., 2002; Araújo et al., 2003; Grallet et al., 2003; Lavinas et al., 2003; Colturato et al., 2005; Abreu et al., 2006; Barboza et al., 2006).

O Centro de Radiofarmácia do IPEN conquistou em 1999 a certificação ISO-9001-2000 para produção e distribuição de radiofármacos por meio de um programa de qualidade aplicado ao IPEN.

Apesar das peculiaridades relacionadas à produção e controle de qualidade de radiofármacos em função da presença do elemento radioativo em sua composição, os mesmos são medicamentos administrados, na maioria das vezes, intravenosamente. Devem, portanto, ser produzidos de acordo com as Boas Práticas de Fabricação (BPF).

Tendo em vista o interesse bastante atual pela regulamentação da produção e uso dos radiofármacos, o presente trabalho pretende rever e compilar os aspectos mais relevantes da aplicação das BPF na produção de radiofármacos, tendo em vista as particularidades de tais preparações, à luz das recomendações já estabelecidas em outros países.

\section{Boas Práticas de Fabricação aplicadas à produção de Radiofármacos}

Os procedimentos regulatórios para controle de produtos radiofarmacêuticos são em grande parte determinados pela origem destes produtos e os métodos de manufatura: radiofármacos preparados em radiofarmácia hospitalar, em radiofarmácia centralizada, em centros e institutos nucleares em escala industrial e em centros de PET (FDA, 1975).

Em todos estes casos, os radiofármacos devem ser manufaturados de acordo com os princípios básicos de Boas Práticas de Fabricação (BPF) para produtos farmacêuticos estéreis conforme recomendado pela OMS.

Boas Práticas de Fabricação (BPF) é um sistema designado para garantir que os fármacos sejam consistentemente produzidos e controlados de acordo com padrões de qualidade, visando eliminar riscos envolvidos na produção. O cumprimento das BPF está direcionado para minimizar os riscos presentes na produção farmacêutica, que não podem ser detectados com a análise do produto final: contaminação cruzada, contaminação com material particulado ou alteração ou mistura de produtos (Agencia Española de Medicamento, 2002; World Health Organization, 1996; Brasil, 2003; Botet, 2006).

A Garantia da Qualidade é um conceito mais amplo que envolve todos os aspectos que, individual ou coletivamente, influenciam na qualidade do produto. Representa a soma de todas as ações realizadas com o objetivo de garantir que os produtos médicos apresentem a qualidade requerida para o uso pretendido. A Garantia da Qualidade incorpora as BPF e o controle de qualidade (Brasil, 2003; Botet, 2006).

Preparados a partir de elementos radioativos que possuem tempo de meia-vida físico específicos (variando de poucos minutos a alguns dias), os radiofármacos possuem prazo de validade geralmente curto, condicionado à meia vida física do elemento radioativo bem como à estabilidade dos compostos, grandemente influenciada pelo efeito de radiólise promovido pela radiação emitida pelo elemento radioativo sobre a própria estrutura do compos- 
to. Os radiofármacos utilizados em PET são os mais críticos por utilizarem radionuclídeos emissores de pósitron de meia vida física ultracurta (inferior a 2 horas), como o flúor-18, com 109 minutos de tempo de meia-vida (Sorenson, Phelps, 1987; Shung, Smith, Tsui, 1992; Chandra, 1992).

Os radiofármacos, devido à meia vida curta, são liberados e administrados aos pacientes pouco tempo após sua produção, e, desta forma, os resultados de certos ensaios de controle de qualidade podem, eventualmente, ser retrospectivos (ex. ensaio de esterilidade, endotoxina, pureza radionuclídica, etc). A implantação de um programa de Garantia da Qualidade é, portanto, essencial (World Health Organization, 2004).

\section{Regulamentos para produção de radiofármacos}

A OMS descreve a Garantia da Qualidade aplicada a produtos farmacêuticos especiais, incluindo radiofármacos, em complemento à documentação já existente disponível para produtos farmacêuticos (World Health Organization, 1996).

As BPF nos EUA fazem parte do "Code of Federal Regulations - CFR", título 21, partes 210 a 226. São publicados pelo FDA como "current Good Manufacturing Practice-cGMP". As partes que se referem especificamente a medicamentos são as partes 210 e 211 .

Compostos radioativos são regulados pelo FDA da mesma forma as outras drogas. O FDA propôs em 1998, adicionar uma nova parte ao CFR 21, a parte 315 , aplicada à aprovação de radiofármacos (drogas e produtos biológicos) para uso in vivo em diagnóstico e monitoração. A regulamentação proposta não se aplica a radiofármacos com finalidade terapêutica. Ao mesmo tempo, o FDA renomeou a sub-parte $\mathrm{D}$ e adicionou as seções 601.30 a 601.35 na parte 601 (CFR 21 parte 601). Estas novas medidas complementaram a regulamentação existente para aprovação de drogas e produtos biológicos nas partes 314 e 601 (CFR21) respectivamente (CFR 21, Part 315 ; CFR 21, Part 601).

De forma ainda complementar, O FDA fornece um guia para condução de inspeções em locais de produção de compostos radioativos para determinar a conformidade com os regulamentos. O programa suplementa os programas de inspeção para produtos estéreis, fornecendo instruções específicas para condução de inspeções em estabelecimentos produtores de radiofármacos.

Compostos de PET são considerados pelo FDA um tipo particular de radiofármacos e, portanto, as Boas Práticas de Fabricação destes compostos foram descritas em procedimento específico (FDA, 2005).
A Comissão Européia adotou em 1991, duas diretivas para regulamentar as BPF na Europa: Diretiva 91/356/EEC substituída pela Diretiva 2003 94/EC, para drogas de uso humano e Diretiva 91/412/EEC, para drogas veterinárias (European Commission, 1991; Agencia Española de Medicamento, 2002; European Commission, 2003).

A Comissão Européia editou um documento que descreve em detalhes a aplicação das BPF na produção de drogas para uso humano e veterinário, tendo como base as Diretrizes citadas. O Anexo 3 deste documento refere-se à produção de radiofármacos, incluindo alguns aspectos relativos à pessoal, instalação e equipamentos, produção e controle de qualidade e distribuição destes produtos (Agencia Española de Medicamento, 2002).

A Associação Européia de Medicina Nuclear (EANM) elaborou um Guia de BPF para produção de radiofármacos. O guia está dividido em duas partes (A e B), considerando os radiofármacos preparados a partir de reagentes liofilizados para marcação (parte A) e os radiofármacos de $\mathrm{PET}$ e outros produzidos localmente (parte B) (European Association of Nuclear Medicine).

Apesar da regulamentação estabelecida nos EUA e Europa possuírem grande influência internacional, dada a importância de tais mercados, muitos países ou grupos de países (ex. MERCOSUL) possuem seus próprios textos que tem peso de lei nos respectivos países.

Alguns países das Américas adotaram o esquema de Certificação da OMS para programa de Garantia da Qualidade e também aplicam procedimentos padrões de BPF na produção local de drogas. Neste sentido, é de grande importância a adoção pela Argentina, Brasil, Chile, Colômbia e México dos princípios de BPF (World Health Organization, 1996).

AArgentina definiu, por meio de uma resolução especial, as Boas Práticas de Preparação Radiofarmacêuticas (ANMAT, 2004).

As BPF no Brasil são publicadas na Resolução RDC 210 de 04 de agosto de 2003, da Agência Nacional de Vigilância Sanitária (ANVISA) do Ministério da Saúde (Brasil, 2003).

No Brasil, até o presente momento, não existe uma regulamentação específica para produção de radiofármacos. Entretanto, duas iniciativas recentes contribuem para esta regulamentação. Em meados de 2006 foi instituída a Subcomissão de Radiofármacos da Farmacopéia Brasileira, incumbida de elaborar as monografias dos radiofármacos. Paralelamente, no final de 2006, a Agência Nacional de Vigilância Sanitária (ANVISA) instituiu um grupo de trabalho para elaborar regulamentação específica para produção e registro de radiofármacos. Outro grupo de trabalho foi também instituído pela ANVISA para regulamentar o uso de tais produtos nos serviços de Medicina Nuclear. 
O IPEN participa ativamente de tais grupos de trabalho, contribuindo desta forma para a regulamentação da produção e uso dos radiofármacos no país.

\section{Programa de Garantia da Qualidade aplicado à produção de radiofármacos}

Alguns aspectos das BPF aplicados ao Programa de Garantia da Qualidade são de especial interesse e devem ser discutidos no contexto da produção de radiofármacos.

\section{Pessoal}

De acordo com as recomendações da OMS, o estabelecimento produtor (hospital, radiofarmácia centralizada, industrial ou centro PET) e seu pessoal devem estar sob controle de uma pessoa com comprovada formação acadêmica, além de demonstrar experiência prática e conhecimentos na área de radiofarmácia e higiene das radiações. O pessoal de suporte técnico e acadêmico deve possuir formação adicional (pós-graduação) ou treinamento técnico e experiência apropriada para execução de suas funções (World Health Organization, 2004).

Todas as pessoas trabalhando nas instalações radiofarmacêuticas, incluindo as de limpeza e manutenção, devem possuir qualificação complementar sobre estes produtos. Em particular, devem receber treinamento em proteção radiológica (Agencia Española de Medicamento, 2002).

Para garantir a segurança da produção de radiofármacos, o pessoal deve ser treinado em BPF, manipulação segura de materiais radioativos e procedimentos de segurança. Ainda podem ser solicitados a realizar cursos periódicos e receber treinamento para atualizar os conhecimentos com base nos últimos desenvolvimentos (World Health Organization, 2004).

\section{Instalações e equipamentos}

Instalações especiais são requeridas para manipulação de elementos radioativos de modo a prevenir a contaminação do meio ambiente por materiais radioativos liberados durante a manipulação e processamento. Nos laboratórios de produção, as atividades processadas são altas, exigindo requerimentos de blindagem e ventilação controlada, sendo ideal o uso de aparatos para manipulação remota (IAEA, 2004).

Para prevenir a liberação descontrolada de contaminação radioativa, o processamento de materiais radioativos requer um sistema fechado, blindado e dotado de exaustão, denominado célula quente ("hot cell") ou cela de processamento. Tais sistemas também podem ser do tipo caixas-de-luvas ("glove-boxes"). As celas são dispostas no laboratório de produção em série, em blocos ou individualmente, sendo dotadas de acesso para manutenção. Dispositivos de controle localizados em frente às celas servem para operar os equipamentos e instrumentos no interior (IAEA, 2004).

Os equipamentos utilizados, incluindo os específicos para medida da radiação, devem ser calibrados e avaliados em intervalos regulares e devem ser conferidos diariamente, ou antes, de iniciar a produção.

A dispensação, embalagem, rotulagem e transporte dos radiofármacos devem obedecer a regulamentações nacionais e internacionais pertinentes (Associação Brasileira de Normas Técnicas, 2005A; 2005B; 2005C; Brasil, 1988; Brasil, 2004; IATA, 2007).

Os rejeitos radioativos devem ser armazenados e controlados. Os sistemas de contenção devem prevenir contaminação e exposição do pessoal dentro e fora das instalações. A eliminação dos resíduos líquidos deve ser monitorada e tratada, enquanto os resíduos sólidos são dispostos em repositórios autorizados para decaimento (IAEA, 2004).

Materiais radioativos são geralmente produzidos em lotes de tamanho pequeno, utilizando materiais em quantidades pequenas. Recipientes de chumbo pesados são utilizados para proteção contra radiação e necessitam de sistema especial para movimentação dentro da área (IAEA, 2004).

Como princípio geral das BPF, os edifícios devem ser localizados, desenhados, construídos, adaptados e mantidos de forma a possibilitar as operações. Laboratórios para manipulação de materiais radioativos devem ser especificamente planejados, levando em consideração aspectos de proteção radiológica além dos aspectos de limpeza e esterilidade (Nash, 2003; World Health Organization, 2004; Jimenez, 2004; Botet, 2006; Zolle, 2007).

A ventilação da instalação produtiva deve atingir requerimentos para prevenir a contaminação de produtos e a exposição do trabalhador à radiação. Padrões de pressão e fluxo de ar adequados devem ser mantidos por métodos apropriados. Os sistemas de ar para as áreas radioativas e não radioativas devem possuir alarmes que permitam verificar a efetividade de ambos os sistemas (Nash, 2003; Jimenez, 2004; Botet, 2006; Zolle, 2007).

Áreas com pressão positiva são requeridas para processamento de produtos estéreis. Em contrapartida, elementos radioativos são geralmente manipulados em ambientes sob pressão negativa. De acordo com as recomendações da OMS, o processamento de radiofármacos poderá ser realizado em ambiente com pressão negativa, rodeado por ambiente de pressão positiva, cuja qualidade requerida do ar seja atingida (Nash, 2003; World Health Organization, 2004; Jimenez, 2004; Botet, 2006; Zolle, 2007). 
Alguns desenvolvimentos recentes no uso de radioisótopos no campo da medicina impactaram significativamente a evolução das instalações e utilidades. A aplicação dos requisitos de BPF para qualidade do ar e condições de processamento nas instalações de manipulação de materiais radioativos tem contribuído com melhorias significativas na construção de celas de processamento e áreas limpas com sistema de ventilação filtrada com HEPA e sistemas de condicionamento de ar (HVAC). Celas para processamento de radiofármacos que empregam conceitos de BPF com classe A (ISO 5) já são disponíveis comercialmente. Apesar disto, a aplicação dos requisitos de áreas limpas nos laboratórios de radioisótopos em geral e celas de processamento em particular, não é uma tarefa fácil (IAEA, 2004).

Devem ser adotadas unidades de ar separadas para as áreas de manipulação de material radioativo e áreas não radioativas. $\mathrm{O}$ ar proveniente do setor de produção radioativo deve passar por filtros apropriados que são regularmente avaliados quanto ao desempenho (Agencia Española de Medicamento, 2002).

As celas construídas para produção de radiofármacos devem atingir os requisitos estabelecidos para um isolador de pressão negativa. As celas devem ser construídas de acordo com padrões técnicos internacionais. As paredes da cela devem ser lisas, impermeáveis, não quebradiças e com cantos arredondados. Deve ser evitada a instalação de componentes permanentes de difícil limpeza (IAEA, 2004).

Aço inox e acrílico são recomendados como material de construção. A superfície de aço inoxidável deve ser polida. As celas devem atingir as recomendações aplicadas para salas de acordo com a regulamentação das BPF (IAEA, 2004).

\section{Condições Assépticas na produção de radiofármacos}

A ocorrência de contaminação cruzada deve ser prevenida pela adoção de sistemas fechados de manufatura (celas), possibilitando a produção de diferentes produtos no mesmo local ou ao mesmo tempo, desde que sejam eficientemente segregados (Nash, 2003; World Health Organization, 2004; Jimenez, 2004; Botet, 2006; Zolle, 2007).

No caso de reagentes liofilizados para marcação, estes devem ser produzidos com procedimento asséptico. De forma geral, deve-se tomar cuidado com a limpeza, esterilização e operação do equipamento de liofilização para uso em produção (World Health Organization, 2004).

De acordo com os requisitos das BPF relativos à produção de fármacos estéreis, os mesmos devem ser produzidos em áreas limpas e a entrada para estas áreas deve ser feita por meio de câmaras de passagem de pessoal e de equi- pamentos/materiais. As áreas limpas devem ser mantidas com padrão de limpeza e fornecimento de ar apropriado, advindo de filtros de eficiência comprovada. Isto protege o produto da contaminação microbiológica do ambiente (Nash, 2003; Jimenez, 2004; Botet, 2006; Zolle, 2007).

No caso das instalações radioativas, estes princípios devem ser incorporados e compatibilizados com os procedimentos de controle de entrada e saída de pessoal de áreas radioativas (controladas) e sistemas de monitoração de eventuais contaminações radioativas (IAEA, 2004).

Os guias sobre BPF são elaborados inicialmente para produtos farmacêuticos convencionais e respectivas condições de produção. Não apresentam detalhes relativos à utilização de materiais radioativos de forma a possibilitar o desenho e operação de instalações radioativas sob condições assépticas (Nash, 2003; Jimenez, 2004; Botet, 2006; Zolle, 2007).

Torna-se necessário estabelecer um documento para harmonizar os princípios de desenho e operação de fármacos radioativos em detalhes já que as soluções aplicadas aos fármacos convencionais nem sempre podem ser diretamente aplicadas aos radiofármacos. As especificações para materiais radioativos são específicas nos seguintes aspectos:

(a) Necessidade de minimizar a dose de radiação das pessoas.

(b) A contaminação radioativa causada pelos materiais deve ser contida e/ou eliminada.

(c) A massa dos ingredientes ativos è extremamente baixa e, portanto, radiofármacos não causam efeito tóxico.

(d) Como os volumes administrados são baixos, os riscos relativos à contaminação microbiológica e pirogênica são reduzidos.

Enquanto os dois primeiros aspectos causam dificuldades significantes na manipulação e na combinação de condições técnicas com os requisitos das BPF, as duas últimas representam vantagens do ponto de vista das BPF (IAEA, 2004).

Algumas soluções harmonizadas já foram propostas para o processamento asséptico de fármacos radioativos, incluindo:

(a) Inserir celas de processamento convencionais (com pressão negativa) em áreas limpas (com pressão positiva), sendo as celas abastecidas com ar filtrado da área limpa ao redor.

(b) Utilizar celas de processamento com controle próprio de fluxo de ar (com ar filtrado e sistema de exaustão), que operam como isoladores em pressão negativa. Estas celas devem ser estanques para prevenir que o ar de fora entre. Estas celas, com grau A ou C, dependendo do tipo de produto, são recomendadas para materiais radioativos, à semelhança dos produtos 
citotóxicos na indústria farmacêutica convencional.

Uma planta de produção de radioisótopos é geralmente representada por grandes salas, equipadas com maquinário pesado (como p. ex. guindastes para levantar e transportar as proteções de chumbo) que geram partículas. Além disto, o tratamento do alvo irradiado inclui várias operações mecânicas que geram material particulado (como por ex, corte da blindagem que reveste o alvo). Além disto, o processamento do alvo envolve operações como adição de ácido e evaporação, altas temperaturas, etc, devendo ser realizadas em ambiente separado ou segregado dos demais processos farmacêuticos de modo a garantir a preservação das condições assépticas. Entende-se por alvo a amostra de material que é especialmente acondicionada para ser bombardeada por nêutrons de um reator nuclear ou partículas carregadas dos aceleradores do tipo ciclotron, originando o material radioativo.

A limpeza e sanitização são aspectos importantes para obtenção de condições assépticas para produção. Contudo, celas de processamento geralmente não podem ser abertas com regularidade para limpeza em virtude das altas taxas de dose a que são expostos os operadores. Apesar de vários agentes de sanitização estarem disponíveis, não há aplicadores eficientes que possibilitem atingir todos os cantos da cela, a menos que peróxido de hidrogênio, álcool isopropílico ou etílico possam ser nebulizados ou aplicados nas superfícies. A eficiência dos métodos de limpeza e sanitização devem ser validadas (Nash, 2003; Jimenez, 2004; Botet, 2006; Zolle, 2007).

\section{Garantia e Controle de Qualidade}

Radiofármacos são geralmente utilizados antes que todos os ensaios de controle de qualidade sejam concluídos, tornando essencial a implantação de um programa de Garantia da Qualidade (World Health Organization, 2004).

A Garantia da Qualidade e/ou Controle de qualidade devem assumir como principais responsabilidades:

(a) A preparação de instruções detalhadas de cada ensaio e análise e a revisão de procedimentos e especificações.

(b) Garantir a identificação e segregação adequada das amostras para prevenir misturas e contaminação cruzada.

(c) Garantir que a monitoração ambiental, qualificação de equipamentos e validação de processos sejam conduzidas conforme apropriado para avaliar a adequação das condições de fabricação.

(d) A liberação ou rejeição de matérias primas, produtos intermediários, materiais de envase e embalagem, e cada lote de preparação final. (e) A avaliação da qualidade e estabilidade dos produtos

finais e, quando necessário, das matérias-primas e produtos intermediários.

(f) O estabelecimento dos prazos de validade, tendo como base o período validado, relacionado a condições específicas de estocagem.

(g) Assumindo a responsabilidade de retenção de amostras de produtos radiofarmacêuticos e gerando registros apropriados da distribuição (World Health Organization, 2004).

Sempre que o tamanho do estabelecimento permitir, a garantia da qualidade e o controle de qualidade devem ser organizados em grupos separados. A Garantia da Qualidade pode ainda incluir a monitoração e validação dos processos produtivos.

O procedimento de amostragem deve ser adaptado aos propósitos da amostragem, tipo de controles a serem realizados, e a natureza do material amostrado (p. ex. um lote de tamanho pequeno e/ou seu conteúdo radioativo). O procedimento deve ser descrito em um protocolo escrito (World Health Organization, 2004).

\section{Validação}

Atenção especial deve ser dada à validação de processos, incluindo validação dos métodos de esterilização, controle de processo e monitoramento dos parâmetros estabelecidos, especialmente do ambiente, particularmente quando o produto necessita ser liberado antes da conclusão dos ensaios de controle, como ocorre com a maioria dos radiofármacos (Agencia Española de Medicamento, 2002; Nash, 2003; World Health Organization, 2004; World Health Organization, 2006).

\section{Controle em processo}

Modernos conceitos de Tecnologia de Análise de Processo ("Process Analytical Technology - PAT") podem ser aplicados, com vantagens, na produção de fármacos e, em especial, na produção de radiofármacos, dada às características peculiares de tais preparações (Nash, 2003; FDA, 2004A).

Os processos de produção de fármacos são geralmente realizados em lotes e amostras são coletadas para avaliar a qualidade de tais lotes. Este procedimento convencional tem sido aplicado, promovendo qualidade e segurança ao público. Contudo, mais recentemente, ferramentas de análise e controle de processo têm sido disponibilizadas, promovendo incremento no desenvolvimento de fármacos, manufatura e garantia da qualidade (FDA, 2004A). 
Ferramentas de controle de processo promovem ganho em qualidade, segurança e/ou eficiência, dependendo do processo ou produto:

(a) Reduzindo os ciclos produtivos pelo uso de medidas e controle em processo.

(b) Prevenindo rejeição ou re-processamento.

(c) Racionalizando o uso de energia e material e aumentando a capacidade produtiva.

(d) Facilitando o processamento contínuo por aumentar a eficiência e controlar variabilidade.

(e) Proporcionando liberação em tempo real.

(f) Aumentando a automação, promovendo a segurança do operador e reduzindo erros humanos (Nash, 2003; FDA, 2004A).

Todos estes ganhos obtidos com a aplicação do conceito PAT podem ser considerados na produção de radiofármacos. Contudo, a melhoria das condições de segurança do operador devido à automação, bem como a liberação em tempo real, são particularmente interessantes, considerando-se, respectivamente, os riscos envolvidos na manipulação de fontes radioativas não seladas e a vida curta dos radiofármacos, normalmente liberados antes dos ensaios de controle de qualidade ser efetivamente concluídos.

\section{CONSIDERAÇÕES FINAIS}

Os princípios de BPF de produtos farmacêuticos estéreis, determinados pela OMS, devem ser estendidos à produção de radiofármacos. Países desenvolvidos, especialmente os Estados Unidos e países da comunidade européia, adotam modelos próprios, baseados nas recomendações da OMS.

No campo dos radiofármacos, é necessário levar em consideração os avanços tecnológicos ocorridos nas últimas décadas, especialmente no campo da manipulação remota e robótica, bem como de controle de processo, já que estas medidas poderão reduzir os níveis de intervenção humana durante o processo de produção e controle de qualidade de radiofármacos (IAEA, 2004).

Paralelamente à inovação tecnológica, automação e controle em processo, a produção de radiofármacos deve permanecer economicamente viável. Desta forma, as instalações de produção de radiofármacos devem não somente sobreviver às normas regulatórias, mas também planejar o crescimento em tais circunstâncias, de modo a atender à crescente demanda de radiofármacos no Brasil.

A recente iniciativa da ANVISA em regulamentar o registro e produção de radiofármacos no país apresenta-se num momento bastante oportuno. A atividade de produção e comercialização de radioisótopos e radiofármacos foi, durante muitos anos, monopólio da Comissão Nacional de
Energia Nuclear (CNEN), exercido por seus Institutos de Pesquisa, particularmente o Instituto de Pesquisas Energéticas e Nucleares (IPEN) em São Paulo e o Instituto de Energia Nuclear (IEN) no Rio de Janeiro. Uma emenda Constitucional promulgada em 2006, entretanto, quebrou o monopólio de produção de radioisótopos e radiofármacos de meia-vida ultracurta (inferior a 2 horas) bem como o de comercialização de radioisótopos e radiofármacos. Neste novo cenário, além dos Institutos da CNEN, produtores tradicionais de radiofármacos, outras instituições públicas ou privadas poderão produzir radiofármacos de meia-vida ultracurta, reforçando a necessidade de regulamentação desta atividade.

A produção de radiofármacos no IPEN cresce a cada ano, atendendo ao crescimento da demanda. A aplicação de radiofármacos tende a crescer ainda mais com a provável distribuição de centros produtores de radiofármacos de meiavida ultracurta por todo o território nacional, estimulados pela quebra do monopólio e pela aplicação de radiofármacos emissores de pósitron como a Fluordeoxiglicose- ${ }^{18} \mathrm{~F}$ no diagnóstico precoce do câncer, controle de recidivas e acompanhamento da eficácia do tratamento. Novos compostos para aplicação em PET e SPECT, bem como para aplicações terapêuticas estão atualmente em fase de desenvolvimento no IPEN e deverão ser introduzidos num futuro próximo na rotina médica nuclear.

A definição e adoção de normas específicas para produção, registro e utilização de radiofármacos no país, bem como a introdução das monografias relativas aos radiofármacos na Farmacopéia Brasileira, são o reflexo da importância e do crescimento constante da aplicação dos radiofármacos e da Medicina Nuclear no cenário de saúde pública nacional, certamente contribuindo para melhoria da qualidade de vida da população brasileira.

\section{ABSTRACT \\ Quality assurance in radiopharmaceutical production}

Radiopharmaceuticals have been produced and distributed in Brazil for almost 40 years, by the Institutes of the Comissão Nacional de Energia Nuclear (CNEN) particularly the Instituto de Pesquisas Energéticas e Nucleares (IPEN), and applied in Nuclear Medicine in diagnostic and therapeutic procedures. Some aspects related to the production, distribution and use of radiopharmaceuticals are very uncommon and different from the conventional drugs, making necessary the introduction of an specific regulation for these radioactive drugs. In this way, the World Health Organization (WHO) 
and health regulatory agencies from different countries have specific legislations to radiopharmaceuticals production and use.

UNITERMS: Radiopharmacy. Radiopharmaceuticals/ production. Radiopharmaceuticals/quality assurance. Radiopharmaceuticals/manufacturing control.

\section{REFERÊNCIAS BIBLIOGRÁFICAS}

ABREU, B.A.L., ARAÚJO, E.B., MENGATTI, J., ABREU, E.A., BRITTO, J.S., ABREU, E.L., MELO, N.A.B., ABREU, J.B. ${ }^{99 \mathrm{~m} T c-D i m e r c a p t o s u c c i n i c ~ a c i d ~(D M S A) ~}$ absolute renal uptake with new kit formulation from Centro de Radiofarmácia - Instituto de Pesquisas Energéticas e Nucleares (IPEN-CNEN). Radiol. Bras., São Paulo, v.39, n.2, p.81, 2006.

AGENCIA ESPAÑOLA DE MEDICAMENTO. Normas sobre Medicamentos de la Unión Europea. Normas de correcta fabricacion, Medicamentos de uso humano y medicamentos veterinarios, editado por Ministerio de Sanidad y Consumo. Madrid: Secretaria General Técnica, 2002, p. 249.

AKANJI, A.G.; MURAMOTO, E.; CALDEIRA FILHO, J.S. COUTO, R.M.; ARAUJO, E.B. Radiolabeling and biodistribution of monoclonal antibody (Mab) anti-CD20 with iodine-131. Braz. Arch. Biol. Technol., Curitiba, v.48, p.6972,2005 .

ANMAT.Administración Nacional de Medicamentos, Alimentos y Tecnologia Médica, Argentina. Disposición 2819-Buenas Practicas de Fabricación de Preparaciones Radiofarmaceuticas - Anexo VII, 2004. Disponível em : $<$ http://www.anmat.gov.ar/normativa/normativa/ medicamentos/disposicion_2004_2819.pdF>.Acesso em: 06 mar. 2007

ARAÚJO, E.B.; CARVALHO, O.G.; MURAMOTO,E.; ALMEIDA,M.A.T.M. Produção de reativos liofilizados para obtenção de L,L-EC-99m Tc. Radiol. Bras., São Paulo, v.28, p.147-150, 1990.

ARAÚJO, E.B. Conjunto de reativos liofilizados de compostos diaminoditiólicos para marcação com tecnécio-99m. Estudo farmacocinético e elaboração de modelos compartimentalizados dos respectivos complexos. São Paulo, 1995, 147p. [Tese de doutorado. Instituto de Pesquisas Energéticas e Nucleares].
ARAÚJO, E.B.; SANTOS, J.S.; COLTURATO, M.T.; MURAMOTO, E.; SILVA, C.P.G. Optimization of a convenient route to produce $\mathrm{N}$-succinimidyl 4radioiodobenzoate for radioiodination of proteins. Appl. Radiat. Isotopes, Oxford, v. 58, p.667-673, 2003.

ARAUJO E.B., NAGAMATI L.T., CALDEIRAFILHO J.S., COLTURATO M.T., SILVA C.P.G. Labeling of DOTATATE with 131-iodine for therapy application. World J. Nucl. Med., Lemesos, v.3, suppl. 1, S32, 2004.

ARAUJO E.B., MURAMOTO, E.; NAGAMATI L.T., CALDEIRAFILHO J.S.; COUTO, R.M.; SILVA, C.P.G. Comparison of ${ }^{131} \mathrm{I}_{-\mathrm{Tyr}^{3} \text {-octreotate and }}{ }^{131}{ }^{\mathrm{I}}$-DOTA-Tyr ${ }^{3}$ octreotate: the effect of DOTA on the pharmacokinetics and stability. INTERNATIONAL SYMPOSIUM ON TRENDS IN RADIOPHRMACEUTICALS (ISTR2005), Vienna, Austria, 2005. Book of extended synopses. Vienna: International Atomic Energy Agency, 2005. p.103-104.

ARAUJO, E.B.; BARBOZA, M.F.; COLTURATO, M.T.; MURAMOTO, E.; HERRERIAS, R.; COUTO, R.M.; MENGATTI, J. Labeling of octreotide with ${ }^{111}$ In using DOTA and DTPA as chelating group: a comparative study. Q. J. Nucl. Med. Mol. Imaging, Milan, v.50, n.1, p.41-42, 2006.

ASSOCIAÇÃO BRASILEIRADENORMAS TÉCNICAS. Referências bibliográficas: NBR 7501. Rio de Janeiro, 2005A. 9p.

ASSOCIAÇÃO BRASILEIRADENORMAS TÉCNICAS. Referências bibliográficas: NBR 7500. Rio de Janeiro, 2005B. 57p.

ASSOCIAÇÃO BRASILEIRADENORMAS TÉCNICAS. Referências bibliográficas: NBR 7503. Rio de Janeiro, 2005C. 10p.

BARBOZA, M.F., FUKUMORI, N.T.O., HERRERIAS,R., SOUZA, A.A., PIRES, J.A., CERQUEIRA FILHO, A., COUTO, R., CAMPOS, D.G., ARAÚJO, E.B., MENGATTI,J., LIMA,E.N. ${ }^{111}$ In-DTPA-Octreotide: production and quality control. Radiol. Bras., São Paulo, v.39, n.2, p. 93, 2006. 
BOTET, J. Boas práticas em instalações e projetos farmacêuticos, tradução e revisão de Julio R. do Amaral, Airton Wilges de Guide pratique pour lês projets d'installations pharmaceutiques. São Paulo: RCN editora, 2006. 360p.

BRAGA, F.J.H.N.; ARAÚJO, E.B.; CAMARGO, E.E. Gallium scintigraphy in Hansen Diseases. Eur. J. Nucl. Méd., Berlin, v.18, p.866-869, 1993A.

BRAGA, F.J.H.N.; ARAÚJO, E.B.; CAMARGO E.E. RIVITTI, M.C.M.; CUCE, L.C. Scintigraphic evaluation of mycetoma. Nucl. Med. Commun., London, v.14, p.814818, 1993B.

BRASIL. Resolução CNEN 13/88. Transporte de Materiais Radioativos. Disponível em: <http://www.cnen.gov.br/ segurança/normas/mostra-norma.asp?op=501>. Acesso em: 15 out. 2007.

BRASIL. Resolução RDC 210, de 04 de agosto de 2003. Regulamento Técnico das Boas Práticas para a Fabricação de Medicamentos, Anexo I, Agência Nacional de Vigilância Sanitária. Disponível em: $<$ http://elegis.anvis a.gov.br/leiref/public/ showAct.php?id=22321\&word=>. Acesso em: 15 out. 2007.

BRASIL. Agência Nacional de Transportes Terrestres (ANTT). Resolução 420 de 2 de fevereiro de 2004. Disponível em: <http://www.antt.gov.br/resolucoes/ 00500/resikycao420_2004.htm>. Acesso em: 15 de out. 2007.

CALDEIRA FILHO, J.S., HERRERIAS, R., BARBOZA, M.F., FUKUMORI, N.T.O., LIMA, E.N., ARAÚJO, E.B. Production of ${ }^{177} \mathrm{Lu}$-DOTA-Tyr ${ }^{3}$-octreotate to clinical application in neuroendocrine tumors. Radiol. Bras., São Paulo, v.39, n.2, p. 96, 2006.

CALDEIRA FILHO J.S., MURAMOTO E., SILVA C.P.G., ARAUJO E.B. Labeling of DOTA-Tyr ${ }^{3}$-octreotate with ${ }^{177} \mathrm{Lu}$-stability and biodistribution study. ALASBIMN J., Santiago, v.7 (28), 2005. Article N AJ28-3. Disponível em: <http:www2.Alasbimnjournal.cl/alasbimn/CDA/ séc b/0,1206,SCID\%253D14142,00.html.> Acesso em: 06 mar. 2007.
CARVALHO, O.G.; ARAÚJO, E.B.; MURAMOTO,E.; ALMEIDA,M.A.T.M. Produção de reativos liofilizados para obtenção de L,L-ECD- ${ }^{99 m}$ Tc. Radiol. Bras., São Paulo, v.29, p.135-137, 1996.

CHANDRA, R. Introdutory Physics in Nuclear Medicine. 4 ed. Philadelphia: Lea \& Febiger, 1992. p. 9-23, 43-53, 141-155, 157-170.

COUTO, R.M., ARAÚJO, E.B., SOUZA, A.A., MENGATTI, J., BARBOZA, M.F. Preparation of Hidroxiapatite $\left({ }^{90} \mathrm{Y}-\mathrm{HA}\right)$ for synovectyomy. Radiol. Bras., São Paulo, v.39, n.2, p. 95, 2006A.

COUTO, R.M., ARAÚJO, E.B., SOUZA, A.A., MENGATTI,J., BARBOZA, M.F. Preparation of ${ }^{90} \mathrm{Y}-$ citrate for synovectyomy. Radiol. Bras., São Paulo, v.39, n.2, p. 95, 2006B.

COLTURATO, M.T.; MURAMOTO, E.; ARAUJO, E.B. Comparative biodistribution profile of ${ }^{131} \mathrm{I}-\mathrm{VIP}$ and ${ }^{131} \mathrm{I}-$ VIP10-28. Braz. Arch. Biol. Technol., v.48, p.79-83, 2005.

CFR-21. US National Archives \& Records Administration Federal Register: Code of Federal Regulations (CFR) Title 21 (Food \& drugs). Disponível em: $<$ www.accessdata.fda.gov/scripts/cfdocs/cfCFR/ CFRSearch.cfm>. Acesso em: 05 mar. 2007.

CFR 21 Part 315 - Code of Federal Regulations, Title 21, volume 5 - Diagnostic Radiopharmaceuticals. Disponível em: $<$ http://www.accessdata.fda.gov/scripts/cdrh/cfdocs/ cfcfr/CFRsearch.cfm?CFRPart=315>. Acesso em: 05 mar. 2007.

CFR 21 Part 601 - Code of Federal Regulations, Title 21, volume 7 - Licensing. Disponível em: $<$ http:// www.accessdata.fda.gov/scripts/cdrh/cfdocs/cfefr/ CFRsearch.cfm?CFRPart=601>. Acesso em: 05 mar. 2007.

\section{EUROPEAN ASSOCIATION OF NUCLEAR MEDICINE} - Guidelines on current Good Radiopharmacy Practice (cGRPP) in the production of radiopharmaceuticals. Disponível em : $<$ http//www.eanm.org/scientific_info/ guidelines $>$. Acesso em: 20 fev. 2007. 
EUROPEAN COMMISSION. Directive 91/ 412/EEC of 23 July 1991, Good manufacturing practice for veterinary medicinal products. Official Journal L228, 17 de agosto de 1991. p.70.

EUROPEAN COMMISSION. Directive 94/EC of 8 October 2003 Good manufacturing practice in of medicinal products for human use and investigational medicinal products for human use. Official Journal L262, 14 de outubro de 2003. p.22-26.

FDA. U.S. Food and Drug Administration. Radioactive drugs and radioactive biological products. Washington, 1975. v. 40, n. 144, Part II.

FDA. U.S. Food and Drug Administration. Guideline on general principles of process validation, 1987. Disponível em: $<$ http://www.fda.gov/cdrh/ode/425,pdf $>$. Acesso em: 05 mar.2007.

FDA. U.S. Food and Drug Administration. Pharmaceutical cGMPs for the $21^{\text {st }}$ Century: A Risk-based approach. Final Report, 2004A. Disponível em: <http://www.fda.gov/ cder/gmp/gmp2004/GMP_finalreport2004.htm>.Acesso em: 05 mar. 2007.

FDA. U.S. Food and Drug Administration. Guidance for Industry: Sterile drug products produced by aseptic processing - Current Good Manufacturing Practice, 2004B. Disponível em: $<$ http://www.fda.gov/cbei/gdlns/ steraseptic.htm>. Acesso em: 05 mar. 2007.

FDA. U.S. Food and Drug Administration. Guidance PET drug products - current good manufacturing practice (CGMP) - draft guidance. U.S. Department of Health and Human Services, Food and Drug Administration, Center for Drug Evaluation and Research (CDER), 2005. Disponível em: <http://www.fda.gov/cder/guidance/ 5425dft2.htm>. Acesso em: 05 mar. 2007.

GRALLERT, S.R.M.; TAVARES, L.C.; ARAÚJO, E.B. Radioligantes para neurorreceptores benzodiazepínicos. Rev. Bras. Ciênc. Farmac., São Paulo, v.39, n.3, p.243254, 2003.

IAEA. TECDOC 1430. Radioisotope handling facilities and automation of radioisotopes production. Viena: IAEA, 2004. $65 \mathrm{p}$.
INTERNATIONAL AIR TRANSPORT ASSOCIATION (IATA). Dangerous Goods Regulations. Resolution 618, Attachment "A". 48 ${ }^{\text {th }}$ ed. Montreal: IATA, 2007. 823p.

JIMENEZ, L. Microbiological contamination control in the pharmaceutical industry. New York: Marcel Dekker, 2004. 310p.

LAVINAS, T.; ARAÚJO, E.B.; SILVA, C.P.G.; CALDEIRA FILHO, J.S.;; SAMPEL, C.J.; MURAMOTO, E. Direct labeling of chemotatic peptide ForNleLFNleYK with radioiodine - in vivo stability evaluation. ALASBIMN J., Santiago, v.6, n.22, 2003. Disponível em: $<$ http:www2.alasbimnjournal.cl/alasbimn/CDA/sec b/ 0,1206,SCID\%253D5999,00.html>.Acesso em: 06 mar. 2007.

NAGAMATI, L.T.; CALDEIRA FILHO, J.S.; MURAMOTO, E.; ARAÚJO, E.B.; DA SILVA, C.P.G. Labeling of DOTA-Tyr3-Octreotate with 131-iodine for therapy application. In: ANNUAL MEETING OF THE SOCIETY OF NUCLEAR MEDICINE, 52., Toronto, 2005. Scientific Abstracts. Toronto: Society of Nuclear Medicine, 2005. n.p., Abstract No 806. (CD-ROM).

NASH, R.A.; WACHTER, A.H. Pharmaceutical process validation. 3 ed. New York: Marcel Dekker, 2003. 860p.

SANTOS, J.S.; COLTURATO, M.T.; MURAMOTO, E.; SILVA, C.P.G.; ARAÚJO, E.B. Radioiodination of proteins using prosthetic group: a convenient way to produce labelled proteins with in vivo stability. Cell. Mol. Biol., Noisy-le-Grand, v.48, n.7, p.735-739, 2002

SHUNG, K.K.; SMITH, M.B.; TSUI, B. Principles of medical imaging. 1 ed. California: Academic Press, 1992. p.164-180, 195-207.

SORENSON, J.A.; PHELPS, M.E. Physics in nuclear medicine. 2 ed. Philadelphia: W.B. Saunders Company, 1987. p. 13-21, 143-151, 391-451, 543-548,

WORLD HEALTH ORGANIZATION. WHO Expert Committee on Specifications for Pharmaceutical Preparation. Thirty-fourth report. Geneva, 1996. 98p. (Technical Report Series 863). 
WORLDHEALTH ORGANIZATION. Radiopharmaceutical products. In: Quality assurance of pharmaceuticals. A compendium of guidelines and related materials. Good Manufacturing practices and inspection. Geneva, 2004. v.2, p. 130-153.

WORLD HEALTH ORGANIZATION. WHO Expert Committee on Specifications for Pharmaceutical Preparation. Fortieth report. Geneva, 2006. 461p. (Technical Report Series 937).
ZOLLE, I. Technetium-99m pharmaceuticals. Preparation and quality control in Nuclear Medicine. Viena: Springer, 2007.345p.

Recebido para publicação em 21 de março de 2007. Aceito para publicação em 27 de setembro de 2007. 